\title{
The human brain: the final journey
}

\author{
Robert T. Knight ${ }^{1}$, Hans-Jochen Heinze ${ }^{2}$ \\ 1 Department of Psychology and Helen Wills Neuroscience Institute, University of California, USA \\ 2 Leibniz Institute for Neurobiology, Otto-von-Guericke University, Magdeburg, Germany \\ Correspondence: rtknight@berkeley.edu
}

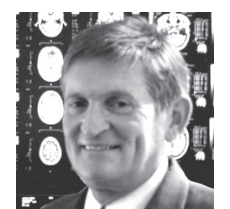

Bob Knight is the Evan Rauch Professor of Neuroscience and Director of the Helen Wills Neuroscience Institute at UC Berkeley. He trained in Neurology at UC San Diego from 1976-1978 and did his postdoctoral work in human neurophysiology at the Salk Institute for Biological Studies from 1978-1980. He was a faculty member in the Neurology Department at UC Davis from 1980-1998 and has been in the Psychology Department and the Helen Wills

Neuroscience Institute at $U C$ Berkeley since 1998. Knight's research focuses on the role of prefrontal cortex in human behavior. His laboratory employs neuropsychological, neuroimaging and electrophysiologocal methods to understand the neural mechanisms underlying frontal cortex control of distributed neuronal ensembles critical for both cognitive and social behavior.
The 'Grand Challenges' of human neuroscience can be simply defined as implementation of all the challenges of our sister journals applied to the human. The task is daunting: To identify the principles that underlie the structural and functional organization of the human brain, to develop strategies against the maladies which affect the human condition and, eventually, to answer the fundamental question of what makes the human brain unique as a cognitive, social and empathetic conscious mind. The last 30 years have seen a veritable explosion of exciting findings in human neuroscience research driven by powerful methodological developments. These advances have transformed human neuroscience from a neuropsychological, lesion-based approach to the current state where sophisticated functional and anatomical analysis is guided by ambitious behavioral theories. While much has been learned, fundamental problems are still waiting to be solved or, worse yet, have not even been defined. For instance, we say that human reasoning, imagination and creativity are forms of information processing in distributed brain networks, but we have no clue how information is represented in the circuits of activated neurons, what coding principles carry information from one brain region to another, and how multiple pieces of information are combined into a coherent entity. This dearth of insight into these fundamentals of neural information processing is illustrated by the fact that we do not even know how the brain accomplishes such a simple task as repeating a spoken word, let alone how the myriads of neural processes that are orchestrated to mediate complex operations in the fields of perception, emotion, social discourse, attention, learning and memory.

While we await enlightenment with respect to the constituting principles of the 'perhaps most complex entity known to science', our knowledge of the anatomical, physiological, chemical and computational aspects of humans brain organization has advanced substantially. This includes critical topics such as how the brain develops and ages across the lifespan. This gain in knowledge will feed into key translational issues addressing how do these normal processes go awry in crippling developmental disorders such as autism or devastating afflictions of aging such as Alzheimer's which eventually robs the person of their own identity. Neuroplasticity is central to all these concerns and the field of human neuroscience needs to begin to take inroads into how the normal human brain enables cognitive and social processing into the clinic. As in all transformative periods of any field 


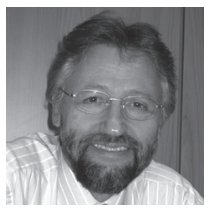

Hans-Jochen Heinze studied medicine and mathematics at the University of Hannover. After attaining his habilitation in 1985 , he worked at the Cognitive Neuroscience Department of the University of California, San Diego, for two years. Back in Hannover, he served as a senior physician and later as the vice chief physician of the Department of Neurology at the medical Faculty in Hannover. In 1993, he became director of the Department of Neurology II at the University of Magdeburg. Since 1997, he is the coordinator of the Center for Advanced Imaging Magdeburg-Bremen. In the Department of Behavioral Neurology, founded in 2005 , his group investigates neural mechanisms underlying human behavior.They successfully integrated the extensively equipped brain-imaging laboratories at Magdeburg into classic neurology, making the Department of Neurology an effective reference center for several nervous diseases. of science, advances in methodology are a key. Accordingly, instead of listing the grand challenges in the massive field of human neuroscience, it might be better to lay out where improved or new methodology might result in paradigm shifts in our understanding of the human brain. Any great result in human behavioral neuroscience will have three key elements: a comprehensive and theory behavioral design, a sophisticated combination of molecular and systemic physiology and a high-resolution identification of neuroanatomical structures engaged in the behavior of interest.

So, there are really three fields of "Grand Challenges" in human neuroscience: Behavioral conceptualization, multi-level physiological integration, and anatomical parcellation. Each element of this triad has multiple aspects, and we will highlight a few. From a behavioral perspective human research has typically followed the standard model in biology: collect a lot of data and sort it out. This is a different model than the more mature field of physics where great empirical work if typically theory driven. The first wave of "modern" human neuroscience emerged in the 1970 driven by the fusion of theory derived by cognitive psychologists coupled with new methods first in human electrophysiology and in the next decade functional magnetic resonance imaging. The current grand challenge is to get the nascent field of theoretical and computational neuroscience talking to experimentalists doing human research. This coupling will lead to new experimental paradigms and novel ways to extract information from the massive data sets being collected in human physiology laboratories. The second grand challenge will be to enhance the spatio-temporal resolution of current physiological research in humans. For instance, can we eventually measure columnar activity non-invasively throughout the cortex as has been shown in early extrastriate cortices? Greater attention also needs to be paid to instances where neuroscientists have an opportunity to study the human brain with implanted electrodes dictated by the clinical condition. It needs to emphasize that the brain is not a set of free floating "modules". Rather, the amazing speed of human processing is dependent on parallel and serial interactions in widespread neural networks. There has been a recent explosion in attempts to employ various methods such as Granger Causality, Dynamic Causal Modeling, Mutual Information Theory to name a few to this key area of research and this work needs to expand and undergo experimental validation. Regarding grand challenge number three we need better in vivo and perhaps in vitro human neuroanatomical methods. Each persons cytoarchitectonic areas are slightly different yet we use a standard stereotactic space to analyze brain activation data. A method to individually parcellate human cytoarchitectonic areas, as has been done for the stripe of Gennari in area 17, would dramatically improve current neuroimaging approaches.

On the bank of the 21th century, human neurosciences are embarking to replace traditional definitions of the human condition with the concept of an evolving brain shaped by natural selection and governed by natural laws. This quest had the additionally "corollary discharge" that understanding the actions of the human mind might eventually be simulated by actions of silicon parts. As put by Dennett, those who fear that this final Galileo challenge will subvert human specialness and cherished values should realize that human dignity is explained, but not destroyed by science: We learn that we are animals, but the only animals who can lead a good life as moral agents.

Front. Neurosci. (2008) 2, 1: 15-16. doi: 10.3389/neuro.01.020.2008

Copyright (C) 2008 Knight. This is an open-access publication subject to an exclusive license agreement between the authors and the Frontiers Research Foundation, which permits unrestricted use, distribution, and reproduction in any medium, provided the original authors and source are credited. 